\title{
Heritability and Genome-wide Association Study To Assess Genetic Differences Between Advanced Age-Related Macular Degeneration Subtypes
}

\author{
Lucia Sobrin, MD, MPH${ }^{1}$, Stephan Ripke, MD², Yi Yu, PhD $^{3}$, Jesen Fagerness, JD2, Tushar \\ R. Bhangale, $\mathrm{PhD}^{4}$, Perciliz L. Tan, BSc ${ }^{5}$, Eric H. Souied, MD, PhD ${ }^{6,7}$, Gabriëlle H.S. \\ Buitendijk, MD, $\mathbf{M S c}^{8}$, Joanna E. Merriam, MD, $\mathrm{PhD}^{9}$, Andrea J. Richardson, BSc ${ }^{10}$, \\ Soumya Raychaudhuri, MD, PhD ${ }^{11}$, Robyn Reynolds, MPH $^{3}$, Kimberly A. Chin, BA ${ }^{3}$, Aaron \\ Y. Lee, MD ${ }^{12}$, Nicolas Leveziel, MD, PhD ${ }^{6,7}$, Donald J. Zack, MD. PhD ${ }^{13,14,15}$, Peter \\ Campochiaro, MD ${ }^{13,14}$, R. Theodore Smith, MD, PhD $^{9}$, Gaetano R. Barile, MD ${ }^{9}$, Ruth E. \\ Hogg, PhD ${ }^{16}$, Usha Chakravarthy, MD, PhD ${ }^{16}$, Timothy W. Behrens, MD ${ }^{17}$, André G. \\ Uitterlinden, $\mathrm{PhD}^{18}$, Cornelia $\mathrm{M}$. van Duijn, $\mathrm{PhD}^{19}$, Johannes R. Vingerling, $\mathbf{M D}, \mathrm{PhD}^{8}$, \\ Milam A. Brantley Jr, MD, PhD ${ }^{12}$, Paul N. Baird, PhD ${ }^{10}$, Caroline C.W. Klaver, MD, PhD $^{8}$, \\ Rando Allikmets, PhD $^{9,20}$, Nicholas Katsanis, PhD $^{5}$, Robert R. Graham, PhD ${ }^{17}$, John P.A. \\ loannidis, MD, DSc ${ }^{21,22,23}$, Mark J. Daly, PhD², and Johanna M. Seddon, MD, ScM ${ }^{3,24}$
}

${ }^{1}$ Department of Ophthalmology, Massachusetts Eye and Ear Infirmary, Boston, Harvard Medical School, Boston, Massachusetts $02114{ }^{2}$ Center for Human Genetic Research, Massachusetts General Hospital, Boston, Massachusetts 02114 and Program in Medical and Population Genetics, Broad Institute, Cambridge, Massachusetts $02142{ }^{3}$ Ophthalmic Epidemiology and Genetics Service, New England Eye Center, Tufts Medical Center, Tufts University School of Medicine, 800 Washington St. \#450, Boston, MA $02111^{4}$ Department of Bioinformatics and Computational Biology, Genentech Inc, South San Francisco, CA $94080{ }^{5}$ Center for Human Disease Modeling and Departments of Cell Biology and Pediatrics, Duke University, Durham, NC $27710^{6}$ Department of Ophthalmology, University Paris Est Creteil, Hopital Intercommunal de Creteil, Creteil, 94000, France ${ }^{7}$ Faculté de Médecine Henri Mondor, Department of ophthalmology. UPEC, Créteil, France ${ }^{8}$ Department Ophthalmology, Department Epidemiology, Erasmus Medical Center, Rotterdam, the Netherlands ${ }^{9}$ Department of Ophthalmology, Columbia University, New York, NY, $10032{ }^{10}$ Centre for Eye Research Australia, University of Melbourne, Royal Victorian Eye and Ear Hospital, East Melbourne, Victoria, Australia ${ }^{11}$ Divisions of Genetics and Rheumatology, Brigham and Women's Hospital, Boston, MA $02115^{12}$ Ophthalmology \& Visual Sciences, Washington University School of Medicine, St Louis, MO 63110, USA and Barnes Retina Institute, St. Louis, MO $63144{ }^{13}$ McKusick-Nathans Institute of Genetic Medicine, Department of Ophthalmology, Wilmer Eye Institute, Johns Hopkins University School of Medicine, Baltimore, MD $21205{ }^{14}$ Department of Neuroscience, Johns Hopkins University School of Medicine, Baltimore, MD $21205{ }^{15}$ Department of Molecular Biology and Genetics and Institut de la Vision, UPMC, Paris, France ${ }^{16}$ Center for Vision and Vascular Science, The Queen's University, Belfast, Northern Ireland. ${ }^{17}$ ITGR Human Genetics Group, Genentech Inc, South San Francisco, CA $94080{ }^{18}$ Department Epidemiology, Department Internal Medicine, Erasmus Medical Center, Rotterdam, the Netherlands ${ }^{19}$ Department Epidemiology, Erasmus Medical Center Rotterdam, the Netherlands ${ }^{20}$ Department of Pathology and Cell Biology, Columbia

Address for reprints: Johanna M. Seddon, MD, ScM, Ophthalmic Epidemiology and Genetics Service, Department of Ophthalmology, Tufts Medical Center, 800 Washington St. \#450, Boston, MA 02111, jseddon@ @uftsmedicalcenter.org, Tel. 617-636-9000, Fax. 617-636-5844.

Conflict of interest: T.R.B, T.W.B. and R.R.G are employees of Genentech Inc. Running Head: Heritability and GWAS of Advanced AMD Subtypes 
University, New York, NY 10032, USA ${ }^{21}$ Department of Hygiene and Epidemiology, University of Ioannina School of Medicine, loannina 45110, Greece ${ }^{22}$ Stanford Prevention Research Center, Department of Medicine, and Department of Health Research and Policy, Stanford University School of Medicine, Stanford, CA 94305, USA; ${ }^{23}$ Center for Genetic Epidemiology and Modeling, ICRHPS, and Tufts Clinical and Translational Science Institute, Tufts Medical Center, Tufts University School of Medicine, Boston, MA 02111, USA; ${ }^{24}$ Department of Ophthalmology, Tufts University School of Medicine, Boston, Massachusetts 02111, USA

\section{Abstract}

Purpose-To investigate whether the two subtypes of advanced age-related macular degeneration (AMD), choroidal neovascularization (CNV) and geographic atrophy (GA), segregate separately in families and to identify which genetic variants are associated with these two subtypes.

Design-Sibling correlation study and genome-wide association study (GWAS)

Participants-For the sibling correlation study, we included 209 sibling pairs with advanced AMD. For the GWAS, we included 2594 participants with advanced AMD subtypes and 4134 controls. Replication cohorts included 5383 advanced AMD participants and 15,240 controls.

Methods-Participants had AMD grade assigned based on fundus photography and/or examination. To determine heritability of advanced AMD subtypes, we performed a sibling correlation study. For the GWAS, we conducted genome-wide genotyping and imputed 6,036,699 single nucleotide polymorphism (SNPs). We then analyzed SNPs with a generalized linear model controlling for genotyping platform and genetic ancestry. The most significant associations were evaluated in independent cohorts.

Main Outcome Measures-Concordance of advanced AMD subtypes in sibling pairs and associations between SNPs with GA and CNV advanced AMD subtypes.

Results-The difference between the observed and expected proportion of siblings concordant for the same subtype of advanced AMD was different to a statistically significant degree ( $\mathrm{P}=4.2 \mathrm{x}$ $10^{-5}$ ) meaning that siblings of probands with CNV or GA are more likely to develop CNV or GA, respectively. In the analysis comparing participants with $\mathrm{CNV}$ to those with GA, we observed a statistically significant association at the ARMS2/HTRA1 locus [rs10490924, odds ratio $\left.(\mathrm{OR})=1.47, \mathrm{P}=4.3 \times 10^{-9}\right]$ which was confirmed in the replication samples $(\mathrm{OR}=1.38, \mathrm{P}=7.4 \mathrm{x}$ $10^{-14}$ for combined discovery and replication analysis).

Conclusions-Whether a patient with AMD develops CNV vs. GA is determined in part by genetic variation. In this large GWAS meta-analysis and replication analysis, the ARMS2/HTRAI locus confers increased risk for both advanced AMD subtypes but imparts greater risk for CNV than for GA. This locus explains a small proportion of the excess sibling correlation for advanced AMD subtype. Other loci were detected with suggestive associations which differ for advanced AMD subtypes and deserve follow-up in additional studies.

\section{Introduction}

Age-related macular degeneration (AMD) is the most common cause of irreversible vision loss in older adults. ${ }^{1}$ Vision loss from AMD most often occurs when the disease progresses to one of two advanced forms, geographic atrophy (GA) or choroidal neovascularization $(\mathrm{CNV})$. Clinical characteristics and histological features of GA and CNV suggest distinct pathological pathways in their development. ${ }^{2-3} \mathrm{GA}$ is usually slowly progressive and involves atrophy of the macular neurosensory cells, whereas $\mathrm{CNV}$ often presents with acute 
vision loss and involves growth of abnormal choroidal vessels under the macula. Both advanced forms can be found in the same patient; GA and CNV can occur in the same eye or a patient may have GA in one eye and CNV in the other. While there are also some predisposing pathophysiologic changes in the extracellular matrix of the macula that are seen in both types of AMD, ${ }^{3}$ it is unclear what leads eyes with early or intermediate disease to progress to one advanced subtype or the other. Some differences in the effects of environmental risk factors, such as hypertension and cigarette smoking, on the development of neovascular vs. non-neovascular AMD have been suggested. ${ }^{4-6}$ However, these environmental risk factors alone do not explain the entire difference in development of the two subtypes.

The genetic predisposition of overall AMD has been clearly demonstrated, ${ }^{1,7}$ and genetic association studies have been very successful in uncovering common variants at several loci that account for this. ${ }^{8-22}$ These case-control association studies have most commonly included a combination of patients with GA and patients with CNV together as advanced cases. Subgroup analyses performed as part of some of these investigations have not generally found significant differences in the risk allele frequencies for $\mathrm{CNV}$ vs. $\mathrm{GA},{ }^{12,15,17-18,20}$ which may be due to limited power given the relatively small number of patients with GA in these investigations. However, in the subgroup analyses for the largest GWAS performed to date and in a candidate gene study performed by our group in a large sample, the ARMS2/HTRAl locus (rs10490924) was associated with a greater risk of CNV compared with GA. ${ }^{21-23}$ Interestingly, no other loci have been proven to predispose specifically to one of the advanced forms. Herein we report new information and expand upon our previous analyses of this subphenotype difference in the following ways: (1) including novel evidence for sibling correlation, (2) investigating more than 6 million additional loci using genome-wide data with improved imputation methods based on the 1000 Genomes Project, ${ }^{24}$ (3) adding in depth analyses of the single nucleotide polymorphisms (SNPs) associated with overall advanced AMD, including the recently reported $F R K / C O L 1 O A 1$ and VEGFA loci, ${ }^{25}$ and (4) incorporating additional replication samples. The purposes of this study were to determine whether development of CNV vs. GA is heritable and to identify genetic variants that may explain differences in risk of CNV vs. GA with an unbiased method of examining genetic loci across the genome.

\section{Methods}

The methods employed in this study conformed to the tenets of the Declaration of Helsinki. Informed consent was obtained from all participants, and this work is Health Insurance Portability and Accountability Act (HIPAA)-compliant. Institutional Review Board (IRB)/ Ethics Committee approval was obtained.

\section{Sibling Correlation - Heritability}

To determine the heritability of GA and CNV, we performed a sibling correlation study. A sibling correlation analysis examines whether siblings are more likely to have the same subtype of advanced AMD than what would be expected by chance alone, if there were no shared genetic or environmental risk factors that contribute to the subtype development. Participants for this analysis were derived from the Family Study of AMD. ${ }^{26-27}$ Families with two or more siblings reported to have AMD (large drusen, GA, or CNV) were recruited from the AMD database of the principal investigator (JMS), from other ophthalmologists throughout the country, from Age-Related Eye Disease Study (AREDS) Ancillary Study, and through various media (newspapers, newsletters, brochures). All siblings were assessed with ocular examination and fundus photography. For this analysis, two siblings were chosen from each family in the Family Study of AMD database. Both siblings had either GA 
or CNV in at least one eye, and if bilateral advanced disease was present, they could not have GA in one eye and CNV in the other eye. If there were only two siblings with advanced AMD in the family that met the criteria, those two were chosen. If the family had more than two siblings with advanced AMD who met the criteria, a computer-generated randomization algorithm selected the two siblings. We calculated the observed proportion of sibling pairs (1) who both had GA as their worse eye grade, (2) who both had CNV as their worse eye grade, and (3) in which one sibling had GA as the worse eye grade and the other sibling had CNV as the worse grade. The expected proportions for these three categories were calculated based on the number of individuals with each eye grade, assuming that the occurrence of the two subphenotypes of CNV and GA was not heritable. The distributions of the observed vs. expected proportions of the three sibling pair categories were compared using the chi-square test.

Because the ARMS2/HTRA1 locus has been shown to contribute to potential heritability of the two subphenotypes, we also calculated the expected proportions for the three sibling pair categories controlling for ARMS2/HTRA1 genotype. For this analysis, participants were divided into three groups based on their observed ARMS2/HTRA1 genotype group (GG, TG and TT). Within each genotype group, we then randomly reassigned each participant's phenotype to $\mathrm{CNV}$ or GA while preserving the observed proportion of participants with CNV vs. GA within each genotype group. This random assignment was performed with a computer-generated algorithm. We then recalculated the proportion of siblings in each category. This analysis was done to assess how much of the sibling correlation we observed for advanced AMD subtype could be explained by ARMS2/HTRA1 genotype.

\section{Genome-wide Association Meta- Analyses}

Discovery Sample Description-For the GWAS, the cases and controls were derived from the Tufts/Massachusetts General Hospital (MGH) GWAS Cohort Study, ${ }^{21}$ the Michigan, Mayo, Age-Related Eye Disease Study (AREDS), Pennsylvania (MMAP) Cohort Study $^{22}$, as well as controls from the Myocardial Infarction Genetics Consortium (MIGen) ${ }^{28}$ and the Genetic Association Information Network (GAIN) Schizophrenia Study. ${ }^{29}$ Information on the publicly available MMAP and GAIN dataset sources is available in Appendix 1 at http://aaojournal.org. All patients were of European ancestry. For the Tufts/MGH GWAS Cohort Study, AMD grade was assigned based on fundus photography and ocular examination (Clinical Age-Related Maculopathy Grading System [CARMS] advanced AMD grades 4 and 5). ${ }^{30}$ CARMS Grade 4 is defined as either GA with involvement of the macular center or noncentral GA at least $350 \mu \mathrm{m}$ in size. CARMS Grade 5 is defined as exudative AMD including nondrusenoid pigment epithelial detachments, serous or hemorrhagic retinal detachments, CNV with subretinal or sub-pigment epithelial hemorrhage or fibrosis, or scarring consistent with the treatment of AMD. If a patient had evidence of both GA and CNV in the same eye, they were classified as a Grade 5 (CNV) in that eye. The final designation of grade for each participant was based on the highest grade in either eye. Therefore a patient had Grade 4 (GA) in one eye and a Grade 5 (CNV) in the other eye, was designated a Grade $5(\mathrm{CNV})$ for the purposes of this study. Examined participants without AMD were unrelated to cases, 60 years of age or older, and were defined as individuals without macular degeneration, categorized as CARMS stage 1, based on fundus photography and ocular examination. We included only MMAP cases with GA or $\mathrm{CNV}$ in the analysis; early and intermediate AMD cases were excluded. MIGen controls have been included in our previous GWAS study and were described in detail. ${ }^{21}$ Shared controls from the GAIN Schizophrenia Study were described in Manolio et al. ${ }^{29}$ The combined Tufts/MGH, MMAP, MIGen and GAIN dataset will be referred to as the Tufts/ MMAP/MIGen/GAIN (TMMG) sample. This dataset has been previously described in further detail. ${ }^{25}$ 
Genotyping-The Tufts/MGH and MIGen samples were genotyped at the Broad Institute and National Center for Research Resources (NCRR) Center for Genotyping and Analysis using the Affymetrix SNP 6.0 GeneChip (AFFY 6.0, 909622 SNPs). ${ }^{31}$ Shared controls from the GAIN study obtained from dbGaP were also genotyped by using the Affymetrix SNP 6.0 GeneChip. MMAP samples obtained from dbGaP were genotyped on the Illumina HumanCNV370v1 Bead Array (ILMN 370, 370404 SNPs). ${ }^{22}$ All samples included in this study met quality-control measures as described previously. ${ }^{21-22}$ For the meta-analysis of GWAS, we included only high quality imputed genotypes (INFO >0.6) and minor allele frequency (MAF) $>0.01 .{ }^{25}$ The INFO score is a measure of imputation reliability. A consensus set of 6,036,699 high quality SNPs from each imputed dataset was analyzed by PLINK using a generalized linear model controlling for the genotyping platform and genetic ancestry based on principal component analysis by EIGENSOFT. The imputed genotypes were coded by the allele dosage for each SNP in the analysis.

GWAS Meta-Analyses-We performed three main GWAS analyses. First, we compared individuals who had CNV in at least one eye to those with GA in at least one eye. If a patient had GA in one eye and CNV in the contralateral eye, they were classified as a CNV case for the primary analyses. Second, we compared individuals who had GA in at least one eye and no CNV in either eye (cases) to individuals without any signs of AMD (controls). Finally we compared individuals who had $\mathrm{CNV}$ in at least one eye (cases) to individuals without any signs of AMD (controls). It is possible that some of these CNV cases had GA in their contraleral eye. We also performed each of these 10 analyses adjusting for age at ascertainment and separately in men and women. To determine if the inclusion of patients who had CNV in one eye and GA in the other eye as CNV cases in the main analyses affected the association results, we performed a sensitivity analysis. The sensitivity analysis consisted of repeating the analysis with the subset of patients with grades available for both eyes and excluding the patients who had CNV in one eye and GA in the other eye. Patients with CNV and GA in the same eye were not excluded for this sensitivity analysis because we did not have sufficiently detailed phenotyping information on most patients to isolate these cases. For novel associations, the P value threshold for significance in the GWAS was $\mathrm{P}=5 \times 10^{-8}$. For SNPs in the thirteen loci that have previously been associated with overall advanced AMD, a $\mathrm{P}$ value threshold for significance of 0.0038 was determined by the Bonferroni correction method.

Replication Samples and Genotyping-Replication of SNPs with the smallest P values from the CNV vs. GA and CNV vs. no AMD analyses was pursued in ten independent cohorts from Johns Hopkins University, Columbia University, Washington University, Genentech, the Rotterdam Study, Centre for Eye Research Australia, an independent replication sample from Tufts/MGH, Hopital Intercommunal de Creteil, the Queen's University of Belfast, and Complications of AMD Prevention Trial. ${ }^{25,32}$ All replication studies applied similar criteria for the diagnosis of cases. SNPs which met genotype quality control criteria in replication cohorts were tested for association with a generalized linear model in PLINK. We used an additive model for each SNP $(0,1$, or 2 minor alleles). We conducted a combined analysis for the results of top SNPs in all participating cohorts using Cochran-Mantel-Haenszel pooling of the odds ratios (OR) and a fixed effects model with inverse-variance weighting.

\section{Results}

\section{Sibling Correlation - Heritability}

From the 390 families that are part of the Family Study of AMD, 209 had sibling pairs who met the inclusion criteria for this sibling correlation analysis. The other 181 families did not 
meet criteria because they did not have two siblings with GA or CNV $(n=145)$ or at least one of the siblings had GA in one eye and CNV in the other eye $(n=36)$. The distribution of sex in the 209 sibling pairs was in line with the overall female predominance in the Family Study of AMD: $15 \%$ were brother-brother pairs, $39 \%$ were sister-brother pairs, and $46 \%$ were sister-sister pairs. The difference between the observed and expected distributions of siblings concordant for the subtype of advanced AMD in their worse eye was statistically significant $\left(\mathrm{P}=4.2 \times 10^{-5}\right)$ (Table 1$)$. This means that if one sibling develops $\mathrm{CNV}$, the other sibling is more likely to develop CNV than GA if they develop advanced AMD. Conversely, if one sibling develops GA, the other sibling is more likely to develop GA than CNV if they develop advanced AMD. This supported the rationale for continuing the analyses to look for genetic variants that exert a differential effect on the risk of GA as compared with CNV.

\section{Genome-wide Association and Replication Analyses}

CNV vs. GA-The TMMG dataset contained 819 participants with GA and 1775 participants with CNV (the discovery cohort). Figure 1 (available at http://aaojournal.org) shows a plot of the first two principal components from this analysis. Principal components analysis is a way to correct for population stratification (allele frequency differences between cases and controls due to systematic ancestry differences) which can cause spurious associations in genetic association studies. ${ }^{33}$ The principal components analysis for genetic association studies takes the 12 genotype data from each individual to infer their genetic ancestry. It plots this genetic ancestry by individual principal components, or axes of genetic variation, to determine if the cases and controls differ in their genetic ancestry. Review of the first two principal components derived from the genetic data show no obvious difference between cases and controls, suggesting absence of any significant population stratification. We plotted our meta-analysis of GWAS P values in Quantile-Quantile (QQ) plots (Figure $2 \mathrm{~A}$ - available at http://aaojournal.org). We observed little statistical inflation in the distribution of the association statistic (inflation factor $\lambda_{\mathrm{gc}}=1.014$ ). Figure $3 \mathrm{~A}$ shows a Manhattan plot for this analysis. In the Manhattan plot, each dot represents one SNP tested in the GWAS. Each dot, or SNP, is plotted according to its location along the chromosomes of the human genome (horizontal axis) and its P value in the GWAS analysis (vertical axis). The higher the dots, the stronger the genetic association. The red line indicates the genomewide significance threshold of $5 \times 10^{-8}$. The ARMS2/HTRAl locus is the only one to meet this significance threshold on the Manhattan plot of the GWAS analysis for CNV vs. GA.

Table 2 includes the most statistically significant SNPs for independent loci from the GA vs. CNV comparison for new and previously reported AMD SNPs. We observed a statistically significant association signal at the ARMS2/HTRAl locus ( $r$ 10490924, $\mathrm{P}=4.3 \times 10^{-9}$ ). Figure 2B (available at http://aaojournal.org) shows the QQ plot of the GWAS meta-analysis results after removal of the ARMS2/HTRAl locus where the line became flattened to $\lambda_{\mathrm{gc}}=0.999$. Removal of the ARMS2/HTRAl locus was done to more easily visualize if there are any other loci that are significantly associated. No other loci were associated at a genome-wide significant $\mathrm{P}$ value $\left(<5 \times 10^{-8}\right)$. However, several variants reached a significance level of 5.5 $\times 10^{-6}$ to $1.4 \times 10^{-6}$ including SNPs in KCTD10 and NFATC2 and SNPs near QSER1, BC017935, LILRA4, NUDT4, RASA1, ADARB2, and UTP18. After adjusting for age at ascertainment, the results were not significantly different, with ARMS2/HTRAl again being the only locus to achieve genome-wide significance $\left(\mathrm{OR}=1.48, \mathrm{P}=3.6 \times 10^{-9}\right)$. None of the other SNPs that have been previously associated with overall AMD were significantly associated in CNV vs. GA analysis.

When we evaluated the contribution of the ARMS2/HTRA1 locus to sibling correlation for AMD subtype, a small amount of the excess sibling correlation could be explained by genetic variation in this region (Table 1). For example, the proportion of sibling pairs 
expected to be concordant for GA assuming no heritability was $7.7 \%$ and the observed proportion was $13.8 \%$, or a $6.1 \%$ difference in the proportion observed vs. expected. This means that there must be shared genetic and/or environmental factors that increase the chance of two siblings having the same subtype of AMD (in this case GA) by 6.1 percentage points over what would be expected if there were no genetic or environmental factors at play. When we controlled for the effect of the ARMS2/HTRAl locus, the proportion of the sibling pairs expected to be concordant for GA expected was $8.1 \%$, which explained $0.4 \%$ ( $8.1 \%$ minus $7.7 \%$ ) of the $6.1 \%$ difference. If two siblings both have at least one $\mathrm{T}$ allele at the ARMS2/HTRA1 locus, they are more likely to both develop CNV than GA although genetic similarity at this locus accounts for a small part of the reason that the siblings are more likely to share the same AMD subtype.

The replication sample consisted of independent datasets not included in the above analyses. These were included in order to confirm the associations detected in the discovery dataset. There were 4515 participants with CNV, 868 participants with GA and 15,240 participants with no AMD in these additional datasets. We examined rs 10490924 further in these samples (Table 3). The direction of the effect for rs10490924 was consistent in most of the replication samples with a final meta-analysis OR of 1.38 [95\% confidence interval (CI) $\left.=1.27,1.51 ; \mathrm{P}=7.4 \times 10^{-14}\right]$. Meta-analysis for the replication datasets alone yielded an OR of $1.32\left(95 \% \mathrm{CI}=1.18,1.48 ; \mathrm{P}=1.8 \times 10^{-6}\right)$. The $\mathrm{T}$ allele at $\mathrm{rs} 10490924$ was associated with a higher risk of CNV compared with GA. When meta-analysis of independent datasets is used to estimate a combined effect, confirmation is needed that the effects found in the individual studies are similar enough that one can be confident that a combined estimate will be a meaningful description of the set of studies. Individual studies' point estimates for the effect of each SNP will vary by chance and some variation is expected. When heterogeneity is present among studies, it means that there is more variation than would be expected by chance alone and combination of these studies using meta-analysis techniques is not valid. There was no evidence for heterogeneity in the meta-analyses for rs 10490924 with P values for $\mathrm{Q}$ test of heterogeneity of $0.07\left(\mathrm{I}^{2}=41.7\right)$ and $0.07\left(\mathrm{I}^{2}=42.7\right)$ for the analyses including and excluding the discovery cohort, respectively. In other words, heterogeneity among studies was not present to a statistically significant degree $(\mathrm{P}<0.05)$.

In the discovery cohort, we also detected a region on chromosome 11 that contained several SNPs in LD with $\mathrm{P}<5 \times 10^{-6}$, including rs4755455 $\left(\mathrm{P}=2.1 \times 10^{-7}\right)$ (Table 2). In age-adjusted analyses, the association with rs 4755455 was also consistent with an $\mathrm{OR}=1.59$

$\left(\mathrm{P}=3.1 \times 10^{-7}\right)$. This association of $\mathrm{rs} 4755455$ with advanced AMD subtype, however, was not consistent across the replication samples, yielding meta-analysis $P$ values of 0.003 and 0.63 for the analyses including and excluding the discovery cohort, respectively (Table 3 ). There was evidence for heterogeneity in the meta-analysis for rs 4755455 with a $\mathrm{P}$ value for $\mathrm{Q}$ test of heterogeneity of $0.007\left(\mathrm{I}^{2}=58.7\right)$. When there is hetereogeneity present in a metaanalysis, one approach is to incorporate it into a random effects model which involves the assumption that the effects being estimated in the different studies are not identical, but follow a distribution. In a random effects meta-analysis, the $\mathrm{P}$ value for the association to rs4755455 was not significant $(\mathrm{P}=0.75)$.

Table 2 also shows the meta-analysis GWAS results comparing participants with GA to participants with CNV for SNPs previously associated with overall advanced AMD. Apart from ARMS2/HTRA1, none of the variants in other loci that have been previously associated with advanced AMD had a statistically significant differential effect on risk of CNV vs. GA. The point estimates of the OR for the comparison of the two subtypes of AMD in the GWAS were $>1.10$ for variants in the C2, CETP, and TIMP3 loci (OR estimates 1.13-1.30). To explore these possible small differences in effect of the genes on CNV vs. GA further, we examined the previously associated loci with additional data, where available, from the 
replication cohorts, and there was no statistically significant association in this larger dataset other than the ARMS2/HTRAl locus association (Table 3). With the additional replication data, $C 2$ and TIMP3 maintained OR > 1.10 but still did not achieve statistical significance.

For the Tufts/MGH dataset, we had bilateral eye grades and were able to perform the additional sensitivity analysis in which we excluded cases with CNV in one eye and GA in the other eye. This dataset included 481 patients with GA and 858 patients who had CNV in at least one eye, of which 221 had CNV in one eye and GA in the other eye. With exclusion of these "mixed" cases from the analysis, there was no significant change in the results apart from a generalized decrease in the $\mathrm{P}$ values due to decreased power expected with removal of $25 \%$ of the CNV cases. For rs 10490924 , the OR was 1.46 (95\% CI=1.24-1.71, P= $\left.4.2 \times 10^{-6}\right)$ including the "mixed" cases and $1.38\left(95 \% \mathrm{CI}=1.16-1.64, \mathrm{P}=2.5 \times 10^{-4}\right)$ excluding the "mixed" cases.

\section{GA vs. no $A M D$ and $C N V$ vs. no $A M D$}

Most highly associated SNPs from GWAS: For the GA vs. no AMD analysis, the TMMG dataset contained 819 participants with GA and 4134 participants without AMD. Figure 3B shows a Manhattan plot for the analysis comparing participants with GA to participants without AMD. As expected, several SNPs previously associated with advanced AMD ( $C F H$, $C F B, A R M S 2 / H T R A 1$ and $C 3$ ) had $\mathrm{P}$ values less than the genome-wide significance threshold of $5 \times 10^{-8}$ as indicated by the red line on the plot. In addition, we detected a region on chromosome 6 that contained three SNPs with $\mathrm{P}<1 \times 10^{-10}$, including rs582301 in the $P E C I$ gene $\left(\mathrm{OR}=4.77, \mathrm{P}=3.2 \times 10^{-11}\right)$. Table 4 lists the most statistically significant SNPs for independent loci from this GWAS analysis; adjustment for age at ascertainment showed similar results. Other than the known genes and PECI, the following variants had $\mathrm{P}$ values that were suggestive of association: rs2842992 (near SOD2) with $\mathrm{P}=3.4 \times 10^{-7}$, rs 1789110 (near $M B P$ ) with $\mathrm{P}=4.2 \times 10^{-7}$, and rs722782 (near $C 8$ orf42) with $\mathrm{P}=1.9 \times 10^{-6}$. Because rs582301 in $P E C I$ was rare and imputed, we pursued direct genotyping in a subset of the sample to try to validate the imputation. In a subset of 21 individuals for whom imputation predicted the genotype AG, only two actually had this genotype on direct genotyping suggesting this association is possibly due to imputation error.

For the CNV vs. no AMD analyses, the TMMG dataset contained 1775 participants with CNV and 4134 participants without AMD. Figure 3C shows a Manhattan plot for the analysis comparing participants with CNV to participants without AMD. Only SNPs that had previously been associated with advanced AMD - CFH, CFI, CFB, ARMS2/HTRAl, and $C 3$ - had $\mathrm{P}$ values less than the genome-wide significance threshold of $5 \times 10^{-8}$ as indicated by the red line on the plot. Table 4 shows the most statistically significant SNPs in independent loci from this analysis. Adjustment for age at ascertainment showed similar results. We detected regions on chromosomes 8 and 18 with several SNPs with $\mathrm{P}<5 \times 10^{-7}$ including rs35691538 (near TRIB1) and rs8091635 (near BRUNOL4) $\left(\mathrm{P}=1.3 \times 10^{-7}\right.$ and $1.7 \times 10^{-7}$, respectively). To further investigate these regions, we attempted replication (Table 3). For rs8091635, the direction of effect was not consistent in the replication sample and, after meta-analysis, the $\mathrm{P}$ value decreased to $2.2 \times 10^{-5}$. There was heterogeneity present in the meta-analysis with $\mathrm{P}=0.008$ for the $\mathrm{Q}$ test $\left(\mathrm{I}^{2}=65.6\right)$. In a random effects metaanalysis, the $\mathrm{P}$ value for the association to rs 8091635 was 0.23 . Because the minor allele of the other SNP rs35691538 is rare and this SNP was not well imputed in replication GWAS datasets, no other replication cohorts with available GWAS data had information to contribute regarding this SNP. Two other variants were suggestive for association with CNV compared with no AMD: rs10262213 (near TRA2A) and rs59795197 (ADAM19) with $\mathrm{P}$ values of $1.6 \times 10^{-6}$ and $3.6 \times 10^{-6}$, respectively. 
Results for SNPs previously associated with overall advanced AMD—Table 5 shows a side-by-side comparison of the results for the GA vs. no AMD and the CNV vs. no AMD comparisons for loci previously associated with overall advanced AMD. We observed significant association signals (below the predetermined threshold for significance of 0.0038 ) for the CNV subtype vs. no AMD comparison at SNPs in loci previously associated with overall advanced AMD. These included $\operatorname{LIPC}\left(\mathrm{P}=2.4 \times 10^{-4}\right)$, TIMP3 $\left(\mathrm{P}=8.0 \times 10^{-5}\right)$, CETP $\left(\mathrm{P}=6.5 \times 10^{-6}\right)$, FRK/COLIOAI $\left(\mathrm{P}=4.1 \times 10^{-5}\right)$, VEGFA $\left(\mathrm{P}=2.0 \times 10^{-4}\right)$, and ABCAl $(\mathrm{P}=0.002)$. For the GA subtype vs. no AMD comparison, we also observed significant association signals at SNPs in many of the loci previously associated with overall advanced AMD. SNPs that met the predetermined threshold for significance of 0.0038 for known SNPs included VEGFA (P=0.002), COL8Al $(\mathrm{P}=0.0036)$ and FRK/COL1OAl $\left(\mathrm{P}=1.4 \times 10^{-4}\right)$. For the ARMS2/HTRAl locus, the OR (95\% CI) for GA vs. no AMD was $2.52(2.23-2.83)$ and the OR $(95 \% \mathrm{CI})$ for $\mathrm{CNV}$ vs. no AMD was 3.67 (3.33, 4.05). Of note, apart from this ARMS2/HTRA1 locus, the magnitude of the odds ratios related to the other genes for the two advanced subtypes were similar with overlapping confidence intervals.

Gender-specific analyses-GWAS analyses were performed for each sex separately for the CNV vs. GA, GA vs. no AMD and CNV vs. no AMD comparisons and did not reveal any additional novel associations. For the ARMS2/HTRAl locus in the CNV vs. GA comparison, the association was similar in both men and women with an $\mathrm{OR}=1.52$ $\left(\mathrm{P}=1.57 \times 10^{-6}\right)$ in the women-only analysis and an $\mathrm{OR}=1.39(\mathrm{P}=0.001)$ in the men-only analysis, showing a larger effect of this locus relative to other genetic loci on CNV vs. GA in both groups.

\section{Discussion}

Data from our sibling analysis provides novel evidence for sibling correlation of the major subphenotypes of AMD - CNV and GA, which can reflect shared genetic and/or environmental risk factors. ${ }^{34-35}$ These results could be used to educate patients who have a family history of one of the advanced forms of AMD about the increased likelihood of developing the same form as their siblings. While this report provides evidence for an increased likelihood for the same advanced AMD subtype among siblings, we do recognize that there can be substantial AMD phenotypic heterogeneity in family members with AMD and it is not uncommon to have several AMD subphenotypes in one family. ${ }^{36}$

Having established excess sibling correlation of these subphenotypes and demonstrated a difference between CNV and GA genetic susceptibility for the ARMS2/HTRAl locus previously, ${ }^{23}$ we undertook an analysis of GWAS data from a larger GWAS meta-analysis to try to uncover additional loci associated with an increased risk of one advanced form over the other. Our aggregate replication cohort sample was the largest to date at the initiation of analyses for this type of subphenotype analysis with $4515 \mathrm{CNV}$ cases, $868 \mathrm{GA}$ cases and 15240 controls with no AMD. We demonstrated a definitive genome-wide significance of the previously suggested increased risk for CNV compared with GA for the $\mathrm{T}$ allele of the ARMS2/HTRAl locus $\left(\mathrm{OR}=1.38, \mathrm{P}=7.4 \times 10^{-14}\right) .{ }^{23,37-39}$ The $\mathrm{T}$ allele at the ARMS2/HTRAl locus has already been well established as a risk factor for overall advanced AMD defined as $\mathrm{GA}$ and/or CNV in either eye. The finding from this paper suggests that while the $\mathrm{T}$ allele increases the risk of developing $\mathrm{CNV}$ and/or GA, it increases the risk of developing CNV to a greater degree than the risk of developing GA in patients who develop advanced AMD. Based on our results $(\mathrm{OR}=1.38)$, for each T allele a participant had at the ARMS2/HTRAI locus, their odds of having CNV in either or both eyes was $38 \%$ higher than their odds of having only GA in either or both eyes at the time of phenotype ascertainment which occurred once they already had advanced AMD (GA or CNV) in at least one eye. Adjusting 
for age at AMD ascertainment showed a similar result. GWAS analyses for each sex separately also demonstrated similar results in men and women.

The patients in this study who were designated CNV cases were primarily patients who only had CNV in one or both eyes and no signs of GA in either eye. However, there were some patients who had CNV in one eye and GA in the other eye. We found that inclusion of these "mixed" participants did not alter the result . Therefore, even in patients who might already have GA in one eye, each T allele they have confers an increased odds of developing CNV in the second eye compared with a similar patient with GA who does not have that allele.

There are some underlying biologic mechanisms which could explain why the ARMS2/ HTRAl locus confers this increased risk for CNV. There is evidence for a role of the HTRA1 protein in the etiology of the angiogenesis involved in $\mathrm{CNV} ;{ }^{40}$ the HTRA1 protein has been localized to vascular endothelium and may favor neovascularization by enhancing degradation of extracellular matrix components or by binding to transforming growth factorbeta, an angiogenic factor. ${ }^{41-42}$ However, in this study the ARMS2/HTRAl locus only accounts for a small amount of the excess sibling correlation for advanced AMD subtype, suggesting there are additional genetic and/or environmental factors, including smoking and nutritional factors, ${ }^{38-39,43}$ predisposing patients to these subtypes.

While commercial genetic testing is currently available for the ARMS2/HTRA1 locus, we do not recommend that this be ordered routinely at this time for AMD patients to determine their odds of developing CNV vs. GA. The contribution to the overall heritability of advanced AMD subtypes conferred by this locus is relatively small and we still do not know all of the genetic and environmental risk factors that contribute to subtype risk. Therefore, genetic testing for ARMS2/HTRA1 alone in the clinical setting would give incomplete insight to a particular patient's risk. As we learn more about additional genetic and environmental risk factors for advanced AMD subtypes, ARMS2/HTRA1 genotype could be part of a comprehensive risk score in clinical practice that would affect monitoring schedules or implementation of preventive treatments. The ARMS2/HTRAl genotype could be used currently in clinical trials for preventive strategies aimed at CNV. Participants with higher risk genotypes for $\mathrm{CNV}$ development could be selected preferentially to improve the power to detect a therapeutic effect, potentially with a shorter follow-up period. ${ }^{44}$

We present the best powered study looking at all the other SNPs that have been definitely associated with overall AMD to date in GWAS. Apart from ARMS2/HTRA1, no other new loci reached genome-wide significance in influencing the risk of one advanced AMD subtype over the other (Table 2). The recently discovered VEGFA polymorphism ${ }^{25}$ was a particularly interesting candidate gene in this analysis given the important role of vascular endothelial growth factor (VEGF) in the pathogenesis of CNV. However, the OR for the VEGFA polymorphism rs4711751 comparing GA to $\mathrm{CNV}$ including the replication samples was $1.06(\mathrm{P}=0.23)$. Two other known loci ( 22 and TIMP3) showed ORs in the $\mathrm{CNV}$ vs. GA analysis that cannot exclude the possibility of small to modest differences in the magnitude of the association of these loci with each AMD subphenotype. We could not confirm a previous report that found $\mathrm{CFH}$ risk alleles were more common in GA cases vs. CNV cases $^{22}$ despite the fact that the current study is much larger and has more power statistically.

There were new variants that had $\mathrm{P}$ values at or near genome-wide significance in the discovery cohort that did not have consistent associations in the independent replication samples: rs4755455 (near QSER1) in the CNV vs. GA analysis and rs8091635 (BRUNOL4) in the CNV vs. no AMD analysis. The inability to replicate these associations could be due to chance statistical fluctuations in the discovery cohort, insufficient power in the replication 
cohort, genotyping/imputation imprecision or heterogeneity between cohorts. Although our sample was the largest to date to evaluate these associations, it was underpowered to detect variants with small effect sizes. To detect an association with a genotype relative risk of 1.10 for one of the AMD subphenotypes with $80 \%$ power, approximately $9,500 \mathrm{CNV}$ and 9,500 GA participants would be needed assuming a $40 \%$ minor allele frequency for the variant (Genetic Power Calculator at pngu.mgh.harvard.edu/ purcell/gpc/, June 8, 2011). In contrast, this large GWAS meta-analysis did have sufficient power to firmly document our previous finding from a candidate gene case-control association study. In that prior study the locus had a moderate effect size with an OR of 1.37 which was quite consistent with the OR of 1.38 observed in this study, ${ }^{23}$ suggesting about a $38 \%$ higher risk of developing CNV vs. GA with each $\mathrm{T}$ allele a participant harbors.

There were several other novel SNPs with association signals whose $\mathrm{P}$ values approached genome-wide significance in the GWAS analyses. For example, rs56209061 in KCTD10 in the CNV vs. GA analysis and rs59795197 in ADAM19 in the CNV vs. no AMD analysis are SNPs that may be related to advanced AMD subtypes and merit follow up in studies with larger datasets.

In summary, we found evidence for genetic susceptibility underlying development of one form of advanced AMD over another. We performed the largest GWAS for advanced AMD subphenotype analysis to date at the onset of the study. We firmly documented the stronger association of the chromosome 10 locus with CNV compared with GA. Several other associated candidate loci for $\mathrm{CNV}$ vs. GA, GA vs. no AMD and CNV vs. no AMD were noted to be worthy of additional follow-up. Larger meta-analysis efforts will be key to increasing power in these subtype analyses and may lead to replication of these loci or identification of additional loci with smaller effect sizes which differ between advanced AMD subtypes.

\section{Supplementary Material}

Refer to Web version on PubMed Central for supplementary material.

\section{Acknowledgments}

Financial Support:

This research was supported in part by an anonymous donor to the research of JMS, grants RO1-EY11309, RO1EY13435, R24-EY017404, and K12-EY16335 from the National Institutes of Health, Bethesda, MD; Massachusetts Lions Eye Research Fund, Inc., New Bedford, MA; Unrestricted grants and Career Development Award from Research to Prevent Blindness, Inc., New York, NY; Foundation Fighting Blindness, Owing Mills, MD; The Macula Vision Research Foundation, West Conshohocken, PA; Kaplen Foundation, Tenafly, NJ; Widgeon Point Charitable Foundation, Armonk, NY; the Alcon Research Institute, Fort Worth, TX; a Fight for Sight postdoctoral award, New York, NY; the National Health \& Medical Research Council of Australia Centre for Clinical Research Excellence \#529923 - Translational Clinical Research in Major Eye Diseases, Canberra, Australia; American Macular Degeneration Foundation, Northampton, MA; and the Macular Degeneration Research Fund of the Ophthalmic Epidemiology and Genetics Service, New England Eye Center, Tufts Medical Center, Tufts University School of Medicine, Boston, MA. CERA receives Operational Infrastructure Support from the Victoria Government. Funding support for NEI-AMD was provided by the National Eye Institute. Funding support for the Genome-Wide Association of Schizophrenia Study was provided by the National Institute of Mental Health (R01 MH67257, R01 MH59588, R01 MH59571, R01 MH59565, R01 MH59587, R01 MH60870, R01 MH59566, R01 MH59586, R01 MH61675, R01 MH60879, R01 MH81800, U01 MH46276, U01 MH46289 U01 MH46318, U01 MH79469, and U01 MH79470).

The funding organizations had no role in the design or conduct of this research. 


\section{References}

1. Seddon, JM.; Sobrin, L. Epidemiology of age-related macular degeneration. In: Albert, DM.; Miller, JW.; Azar, DT.; Blodi, B., editors. Albert \& Jakobiec's Principles and Practice of Ophthalmology. 3rd ed. Vol. vol. 1. Philadelphia, PA: Saunders; 2008. p. 413-422.

2. de Jong PT. Age-related macular degeneration. N Engl J Med. 2006; 355:1474-1485. [PubMed: 17021323]

3. Zarbin MA. Current concepts in the pathogenesis of age-related macular degeneration. Arch Ophthalmol. 2004; 122:598-614. [PubMed: 15078679]

4. Seddon JM, Willett WC, Speizer FE, Hankinson SE. A prospective study of cigarette smoking and age-related macular degeneration in women. JAMA. 1996; 276:1141-1146. [PubMed: 8827966]

5. Hyman L, Schachat AP, He Q, Leske MC. Age-Related Macular Degeneration Risk Factors Study Group. Hypertension, cardiovascular disease, and age-related macular degeneration. Arch Ophthalmol. 2000; 118:351-358. [PubMed: 10721957]

6. Complications of Age-related Macular Degeneration Prevention Trial (CAPT) Research Group. Risk factors for choroidal neovascularization and geographic atrophy in the Complications of AgeRelated Macular Degeneration Prevention Trial. Ophthalmology. 2008; 115:1474-1479. [PubMed: 18502512]

7. Haddad S, Chen CA, Santangelo SL, Seddon JM. The genetics of age-related macular degeneration: a review of progress to date. Surv Ophthalmol. 2006; 51:316-363. [PubMed: 16818082]

8. Klein RJ, Zeiss C, Chew EY, et al. Complement factor H polymorphism in age-related macular degeneration. Science. 2005; 308:385-389. [PubMed: 15761122]

9. Hageman GS, Anderson DH, Johnson LV, et al. A common haplotype in the complement regulatory gene factor $\mathrm{H}(\mathrm{HF} 1 / \mathrm{CFH})$ predisposes individuals to age-related macular degeneration. Proc Natl Acad Sci U S A. 2005; 102:7227-7232. [PubMed: 15870199]

10. Haines JL, Hauser MA, Schmidt S, et al. Complement factor H variant increases the risk of agerelated macular degeneration. Science. 2005; 308:419-421. [PubMed: 15761120]

11. Edwards AO, Ritter R III, Abel KJ, et al. Complement factor $\mathrm{H}$ polymorphism and age related macular degeneration. Science. 2005; 308:421-424. [PubMed: 15761121]

12. Rivera A, Fisher SA, Fritsche LG, et al. Hypothetical LOC387715 is a second major susceptibility gene for age-related macular degeneration, contributing independently of complement factor $\mathrm{H}$ to disease risk. Hum Mol Genet. 2005; 14:3227-3236. [PubMed: 16174643]

13. Yang Z, Camp NJ, Sun H, et al. A variant of the HTRAl gene increases susceptibility to agerelated macular degeneration. Science. 2006; 314:992-993. [PubMed: 17053109]

14. Dewan A, Liu M, Hartman S, et al. HTRAl promoter polymorphism in wet age-related macular degeneration. Science. 2006; 314:989-992. [PubMed: 17053108]

15. Jakobsdottir J, Conley YP, Weeks DE, et al. Susceptibility genes for age-related maculopathy on chromosome 10q26. Am J Hum Genet. 2005; 77:389-407. [PubMed: 16080115]

16. Gold B, Merriam JE, Zernant J, et al. AMD Genetics Clinical Study Group. Variation in factor B $(B F)$ and complement component $2(C 2)$ genes is associated with age-related macular degeneration. Nat Genet. 2006; 38:458-462. [PubMed: 16518403]

17. Maller J, George S, Purcell S, et al. Common variation in three genes, including a noncoding variant in $\mathrm{CFH}$, strongly influences risk of age-related macular degeneration. Nat Genet. 2006; 38:1055-1059. [PubMed: 16936732]

18. Maller JB, Fagerness JA, Reynolds RC, et al. Variation in complement factor 3 is associated with risk of age-related macular degeneration. Nat Genet. 2007; 39:1200-1201. [PubMed: 17767156]

19. Yates JR, Sepp T, Matharu BK, et al. Genetic Factors in AMD Study Group. Complement C3 variant and the risk of age-related macular degeneration. N Engl J Med. 2007; 357:553-561. [PubMed: 17634448]

20. Fagerness JA, Maller JB, Neale BM, et al. Variation near complement factor I is associated with risk of advanced AMD. Eur J Hum Genet. 2009; 17:100-104. [PubMed: 18685559]

21. Neale BM, Fagerness J, Reynolds R, et al. Genome-wide association study of advanced age-related macular degeneration identifies a role of the hepatic lipase gene (LIPC). Proc Natl Acad Sci U S A. 2010; 107:7395-7400. [PubMed: 20385826] 
22. Chen W, Stambolian D, Edwards AO, et al. Complications of Age-Related Macular Degeneration Prevention Trial (CAPT) Research Group. Genetic variants near TIMP3 and high- density lipoprotein-associated loci influence susceptibility to age-related macular degeneration. Proc Natl Acad Sci U S A. 2010; 107:7401-7406. [PubMed: 20385819]

23. Sobrin L, Reynolds R, Yu Y, et al. ARMS2/HTRA1 locus can confer differential susceptibility to the advanced subtypes of age-related macular degeneration. Am J Ophthalmol. 2010; 151:345352. [PubMed: 21122828]

24. Marth GT, Yu F, Indap AR, et al. 1000 Genomes Project. The functional spectrum of lowfrequency coding variation. Genome Biol. 2011; 12:R84. Available at: http://genomebiology.com/ content/pdf/gb-2011-12-9-r84.pdf. [PubMed: 21917140]

25. Yu Y, Bhangale TR, Fagerness J, et al. Common variants near FRK/COL1OA1 and VEGFA are associated with advanced age-related macular degeneration. Hum Mol Genet. 2011; 20:36993709. [PubMed: 21665990]

26. Seddon JM, Santangelo SL, Book K, et al. A genomewide scan for age-related macular degeneration provides evidence for linkage to several chromosomal regions. Am J Hum Genet. 2003; 73:780-790. [PubMed: 12945014]

27. Sobrin L, Maller JB, Neale BM, et al. Genetic profile for five common variants associated with age-related macular degeneration in densely affected families: a novel analytic approach. Eur J Hum Genet. 2010; 18:496-501. [PubMed: 19844262]

28. Myocardial Infarction Genetics Consortium. Genome-wide association of early-onset myocardial infarction with single nucleotide polymorphisms and copy number variants. Nat Genet. 2009; 41:334-341. [PubMed: 19198609]

29. GAIN Collaborative Research Group. New models of collaboration in genome-wide association studies: the Genetic Association Information Network. Nat Genet. 2007; 39:1045-1051. [PubMed: 17728769]

30. Seddon JM, Sharma S, Adelman RA. Evaluation of the clinical age-related maculopathy staging system. Ophthalmology. 2006; 113:260-266. [PubMed: 16458093]

31. Korn JM, Kuruvilla FG, McCarroll SA, et al. Integrated genotype calling and association analysis of SNPs, common copy number polymorphisms and rare CNVs. Nat Genet. 2008; 40:1253-1260. [PubMed: 18776909]

32. Complications of Age-Related Macular Degeneration Prevention Trial Study Group. The Complications of Age-Related Macular Degeneration Prevention Trial (CAPT): rationale, design and methodology. Clin Trials. 2004; 1:91-107. [PubMed: 16281465]

33. Price AL, Patterson NJ, Plenge RM, et al. Principal components analysis corrects for stratification in genome-wide association studies. Nat Genet. 2006; 38:904-909. [PubMed: 16862161]

34. Seddon JM, Ajani UA, Mitchell BD. Familial aggregation of age-related maculopathy. Am J Ophthalmol. 1997; 123:199-206. [PubMed: 9186125]

35. Klaver CC, Wolfs RC, Assink JJ, et al. Genetic risk of age-related maculopathy: population-based familial aggregation study. Arch Ophthalmol. 1998; 116:1646-1651. [PubMed: 9869796]

36. De la Paz MA, Pericak-Vance MA, Haines JL, Seddon JM. Phenotypic heterogeneity in families with age-related macular degeneration. Am J Ophthalmol. 1997; 124:331-343. [PubMed: 9439359]

37. Shuler RK Jr, Schmidt S, Gallins P, et al. Phenotype analysis of patients with the risk variant LOC387715 (A69S) in age-related macular degeneration. Am J Ophthalmol. 2008; 145:303-307. [PubMed: 18061132]

38. Seddon JM, Reynolds R, Maller J, et al. Prediction model for prevalence and incidence of advanced age-related macular degeneration based on genetic, demographic, and environmental variables. Invest Ophthalmol Vis Sci. 2009; 50:2044-2053. [PubMed: 19117936]

39. Seddon JM, Francis PJ, George S, et al. Association of CFH Y4O2H and LOC387715 A69S with progression of age-related macular degeneration. JAMA. 2007; 297:1793-1800. [PubMed: 17456821]

40. Jones A, Kumar S, Zhang N, et al. Increased expression of multifunctional serine protease, HTRA1, in retinal pigment epithelium induces polypoidal choroidal vasculopathy in mice. Proc Natl Acad Sci U S A. 2011; 108:14578-14583. [PubMed: 21844367] 
41. Ding X, Patel M, Chan CC. Molecular pathology of age-related macular degeneration. Prog Retin Eye Res. 2009; 28:1-18. [PubMed: 19026761]

42. An E, Sen S, Park SK, et al. Identification of novel substrates for the serine protease HTRA1 in the human RPE secretome. Invest Ophthalmol Vis Sci. 2010; 51:3379-3386. [PubMed: 20207970]

43. Ho L, van Leeuwen R, Witteman JC, et al. Reducing the genetic risk of age-related macular degeneration with dietary antioxidants, zinc, and omega-3 fatty acids: the Rotterdam Study. Arch Ophthalmol. 2011; 129:758-766. [PubMed: 21670343]

44. Seddon JM, Reynolds R, Yu Y, et al. Risk models for progression to advanced age related macular degeneration using demographic, environmental, genetic, and ocular factors. Ophthalmology. 2011; 118:2203-2211. [PubMed: 21959373] 


\section{Table 1}

Sibling correlation in advanced age-related macular degeneration (AMD) subtypes

\begin{tabular}{lllll}
\hline $\begin{array}{l}\text { Siblings' Worse } \\
\text { Eye Grade }\end{array}$ & $\begin{array}{l}\text { Number } \\
\text { Observed }\end{array}$ & $\begin{array}{l}\text { Proportion } \\
\text { Observed }\end{array}$ & $\begin{array}{l}\text { Proportion } \\
\text { Expected }\end{array}$ & $\begin{array}{l}\text { Proportion } \\
\text { Expected } \\
\text { Controlling for } \\
\text { ARMS2/HTRAI } \\
\text { genotype }\end{array}$ \\
\hline Both siblings GA & 29 & $13.8 \%$ & $7.7 \%$ & $8.1 \%$ \\
One sibling CNV, One sibling GA & 58 & $27.8 \%$ & $40.0 \%$ & $38.8 \%$ \\
Both siblings CNV & 122 & $58.4 \%$ & $52.3 \%$ & $53.1 \%$ \\
\hline & & & $\mathrm{P}=4.2 \times 10^{-5}$ & \\
\end{tabular}

$\mathrm{GA}=$ geographic atrophy, $\mathrm{CNV}=$ choroidal neovascularization

The $\mathrm{P}$ value for the chi square test was calculated by comparing the difference between the observed and expected distributions of siblings concordant for the subtype of advanced AMD in their worse eye. The expected distributions were calculated assuming no heritability of the two subphenotypes of CNV and GA and based solely on the number of individuals with each eye grade. 


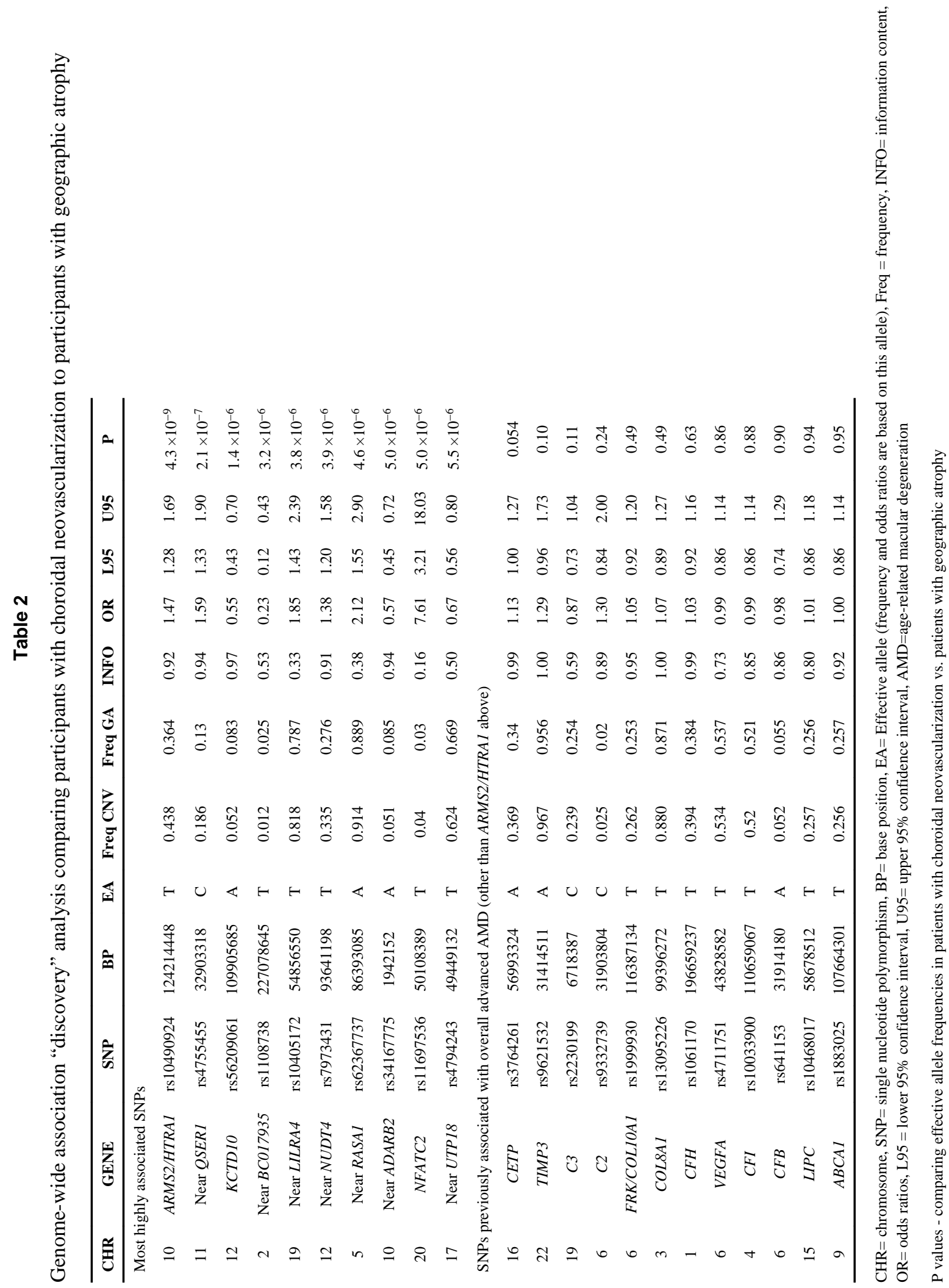




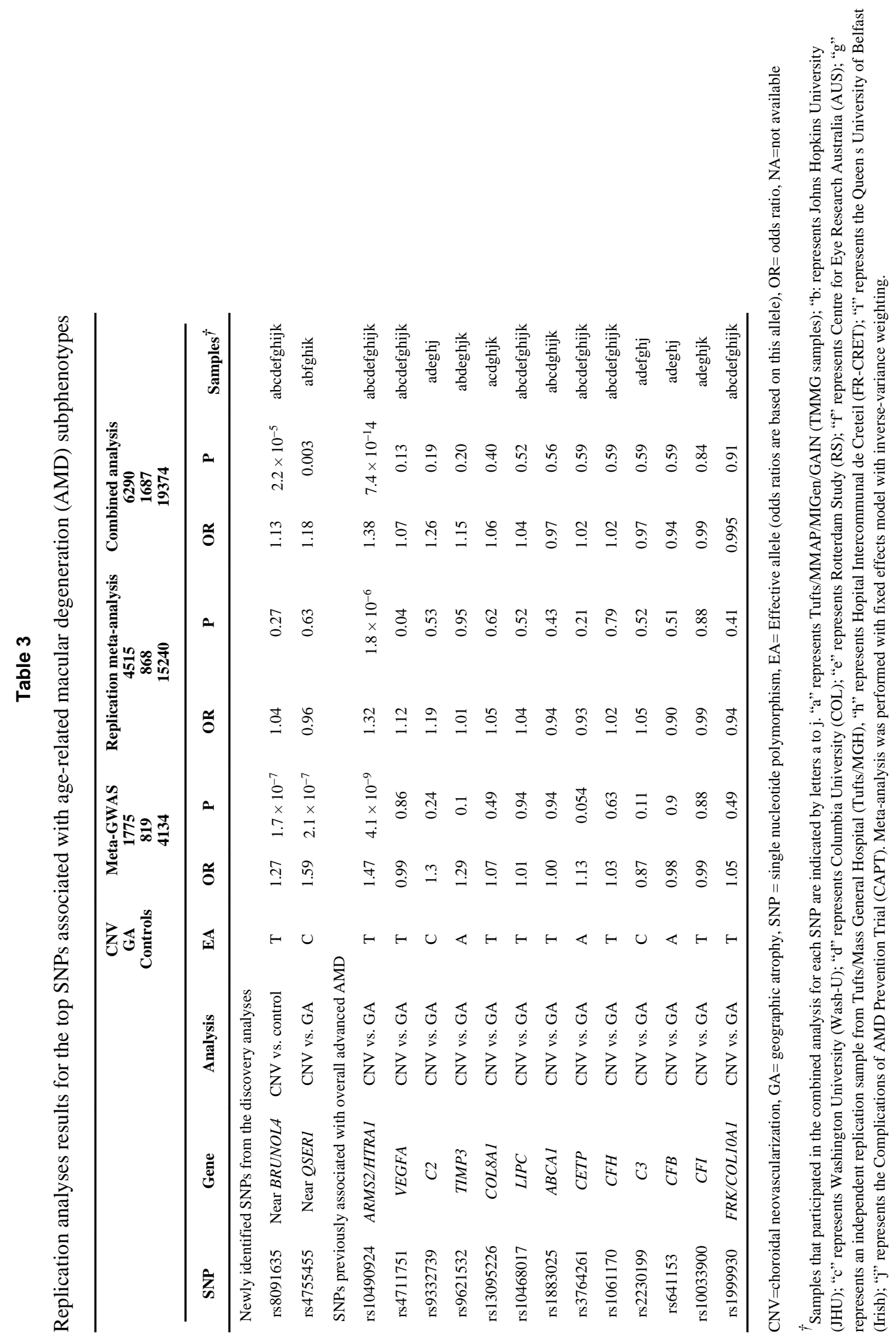




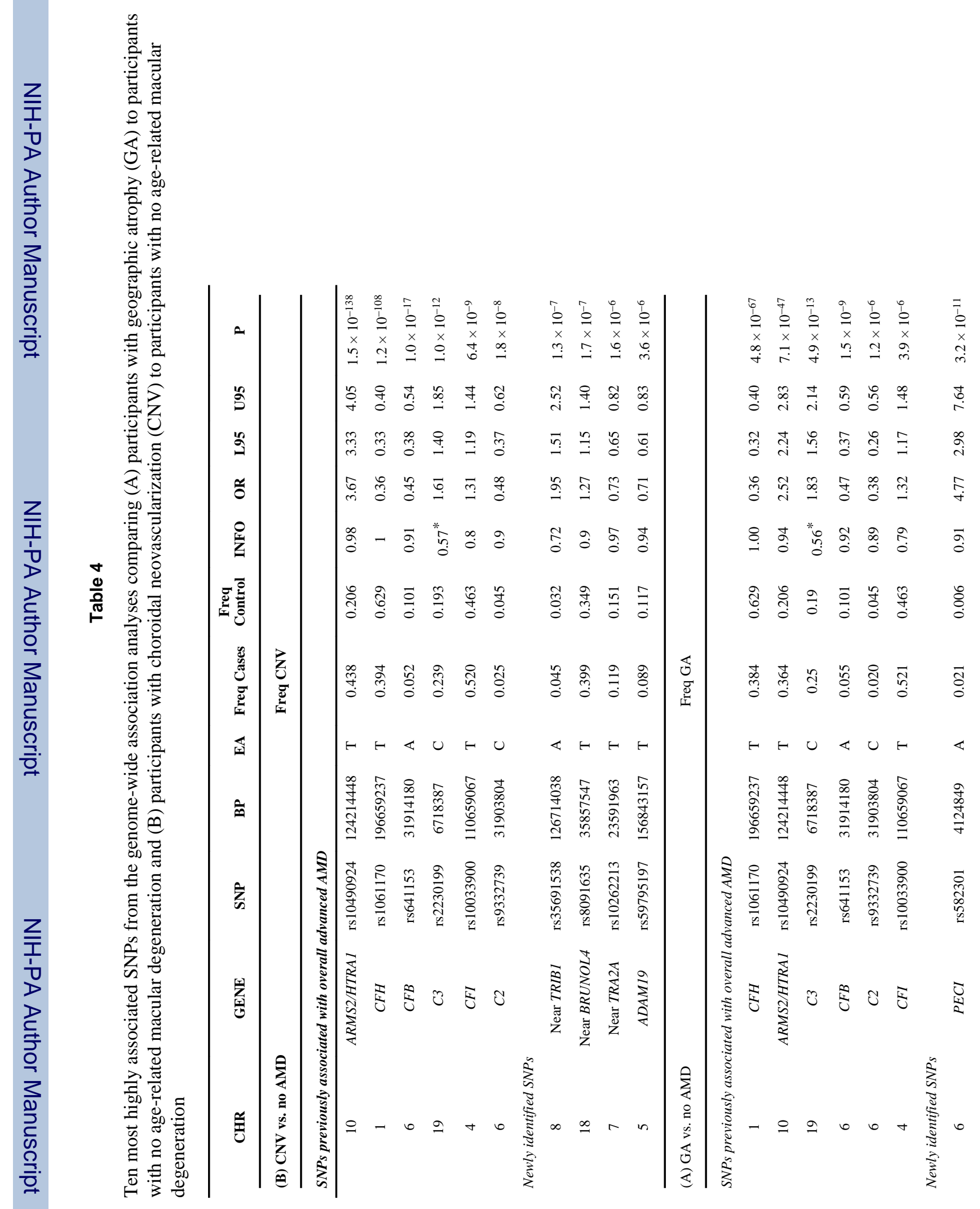




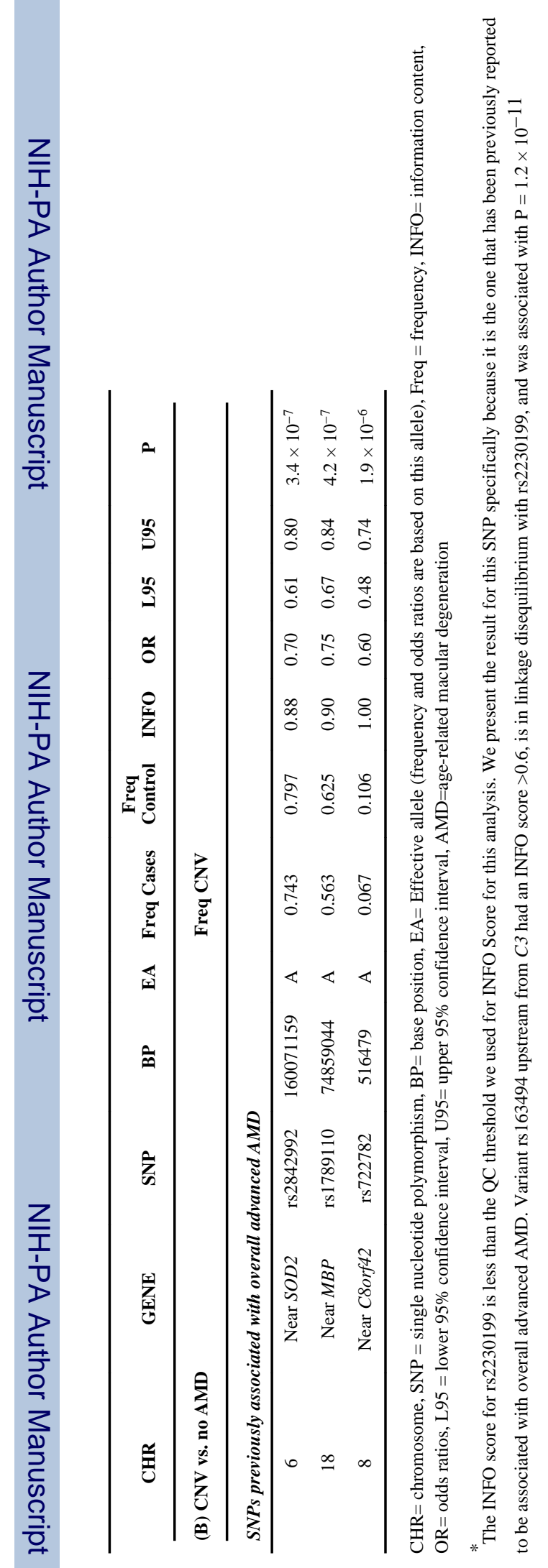

Ophthalmology. Author manuscript; available in PMC 2014 January 23. 


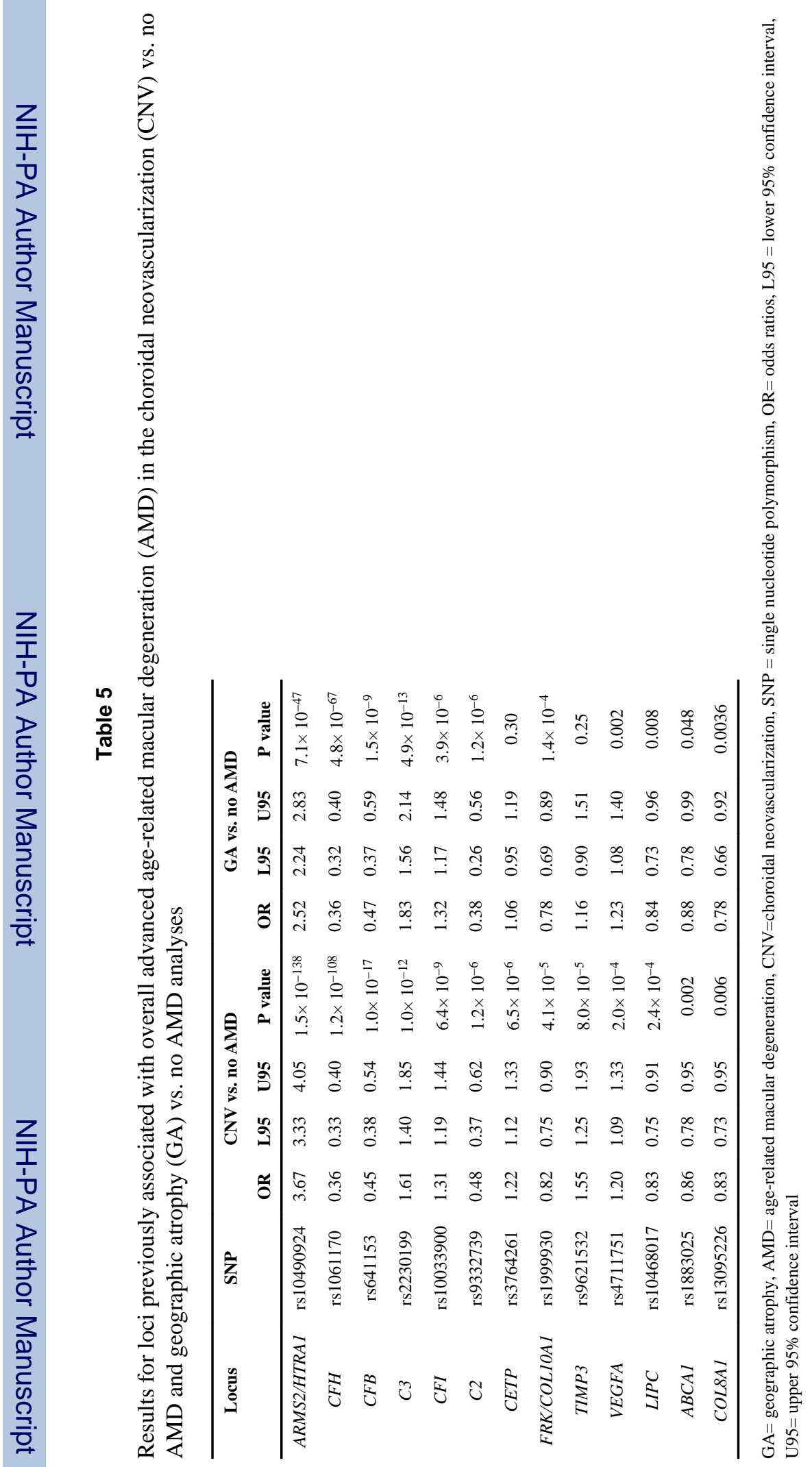

